\title{
Venous Chimney Procedure: A Novel Technical Solution to Prevent latrogenic Budd-Chiari Syndrome Following Retrohepatic Vena Cava Injury
}

\author{
Dan Hebron ${ }^{1}$, Offer Galili², Boris Kessel ${ }^{3}$, Itay Zoarets ${ }^{4}$, Yoram Klein ${ }^{5}$, \\ Aiya Assif ${ }^{6}$ and Eitan Heldenberg ${ }^{7}$ \\ 'Invasive Radiology Unit, Hillel Yaffe Medical Center, Hadera, affiliated to the Bruce Rapaport Faculty of Medicine, \\ Technion - Israel Institute of Technology, Haifa, Israel \\ 2Department of Vascular Surgery, Carmel Medical Center, Haifa, affliated to the Bruce Rapaport Faculty of Medicine, \\ Technion - Israel Institute of Technology, Haifa, Israel \\ ${ }_{3}^{3}$ Trauma Unit, Hillel Yaffe Medical Center, Hadera, affiliated to the Bruce Rapaport Faculty of Medicine, \\ Technion - Israel Institute of Technology, Haifa, Israel \\ ${ }^{4}$ Trauma Unit, Assuta Medical Center, Ashdod, affiliated to the Goldman Faculty of Medicine, Ben Gurion University, \\ Beer Sheva, Israel \\ ${ }^{5}$ Trauma Unit, Sheba Medical Center, Ramat Gan, affliated to the Sackler Faculty of Medicine, Tel Aviv University, Tel Aviv, Israel \\ ${ }^{6}$ Cardiology Division, Hillel Yaffe Medical Center, Hadera, affiliated to the Bruce Rapaport Faculty of Medicine, \\ Technion - Israel Institute of Technology, Haifa, Israel \\ 'Department of Vascular Surgery, Hillel Yaffe Medical Center, Hadera, affiliated to the Bruce Rapaport Faculty of Medicine, \\ Technion - Israel Institute of Technology, Haifa, Israel
}

\begin{abstract}
Retro hepatic IVC injuries (RHVCI) are extremely rare and lethal. The open surgical technique of treating these injuries is a master skill which is not available for most surgeons taking care of these patients. The endovascular trauma management (EVTM) application dictates a new approach in some cases based on applying arterial treatment concepts to the venous trauma cases.

We hereby describe a novel technique in which the known chimney technique, often used to treat juxta renal abdominal aortic aneurysm, was used to prevent iatrogenic Budd Chiary which might have been caused by hepatic veins drainage occlusion by a Stent Graft (SG) that was inserted to treat RHVCl.

Care should be taken to prevent secondary cardiac injury by long SG.
\end{abstract}

Keywords: Penetrating Injury; Retrohepatic Vena Cava; Hepatic Veins; Endovascular Treatment; Stenting

Received: 1 May 2020; Accepted: 4 September 2020

\section{INTRODUCTION}

Traumatic inferior vena cava (IVC) lesions account for approximately $25 \%$ of abdominal vascular injuries.

\section{Corresponding author:}

Eitan Heldenberg, Department of Vascular Surgery, Hillel Yaffe Medical Center, Ha-Shalom Street, P.O.B. 169, Hadera, 3810101, Israel.

Email: eitan@heldenberg.name

(C) 2020 CC BY 4.0 - in cooperation with Depts. of Cardiothoracic/ Vascular Surgery, General Surgery and Anesthesia, Örebro University Hospital and Örebro University, Sweden
These injuries are among the most challenging and lethal lesions sustained by trauma patients, with an overall mortality rate of up to $92 \%$. As many as $50 \%$ of casualties die before reaching medical care, and for those who reach trauma centers, the mortality is between $20 \%$ and $57 \%$ [1].

Retrohepatic vena cava (RHVC) injuries (RHVCI) are sporadic, and therefore both the treating trauma surgeon and the vascular surgeon often lack the necessary experience to deal with such complicated injuries. The mortality rates secondary to these injuries are incredibly high. Improving the outcome of these injuries remains a significant challenge to modern trauma care $[2,3]$. 
The treatment of RHVCI confronts the treating surgeon with major obstacles due to the anatomic location of the RHVC at the posterior aspect of the liver and the abundancy of bridging veins between the RHVC and the liver. These anatomic obstacles create a significant technical challenge in gaining proximal and distal control, in proximity to the injured RHVC, frequently both abdominal and thoracic exposure.

The average trauma surgeon, as well as the vascular surgeon, lack the ability to deal with complex injuries due to their decreasing experience with open surgery, especially for vascular surgeons who are mainly familiar with endovascular techniques [4].

The advancements in endovascular techniques have introduced new treatment alternatives. Exploration of a retro-hepatic hematoma, which might be the single thing that prevents free venous bleeding, should be avoided. Venous balloon occlusion might be used as a bridging maneuver, for proximal and distal control, during hybrid repair. Our unique case highlights the option of total endovascular treatment of this extremely challenging venous injury.

\section{CASE DESCRIPTION}

A previously healthy 22 -year-old male was shot in his right upper abdomen. On scene, the patient was found to be slightly tachycardic, with a heart rate of $112 \mathrm{bpm}$, and had diminished breathing sounds over the right lung. The rest of his physical examination was unremarkable.

Upon arrival at the trauma bay, a chest tube was inserted to the right pleural space draining $600 \mathrm{ml}$ of dark venous blood. On physical examination, an entrance wound was noted below the right rib cage on the midclavicular line and the exit wound was found at the T10 vertebral level. Neurologic examination revealed that the patient was paraplegic. A chest computerized tomography (CT) scan revealed small residual pneumohemothorax. An abdominal CT scan demonstrated grade 5 liver injury with a surrounding large hematoma, retrohepatic vena cava (RHVC) tear, and left hepatic vein laceration (Figure 1).

Due to hemodynamic instability, an emergency laparotomy was conducted. Although the liver was tightly packed, following evacuation of 1.51 of blood from the peritoneal cavity and extensive blood products transfusion, the patient's hemodynamic status did not improve. In order to treat the RHVCI, a 12F sheath (Medtronic Sentrant ${ }^{\mathrm{TM}}$, USA) was inserted percutaneously to the right common femoral vein, through which a compliant balloon (Medtronic Reliant ${ }^{\mathrm{TM}}$, USA) was inserted and inflated at the RHVC level using C-arm fluoroscopic guidance (Figure 2).

The balloon inflation stabilized the patient's blood pressure and an attempt of liver packing removal was performed. An expansion of the RHVC hematoma to the diaphragm level necessitated liver repacking. A $34 \times$

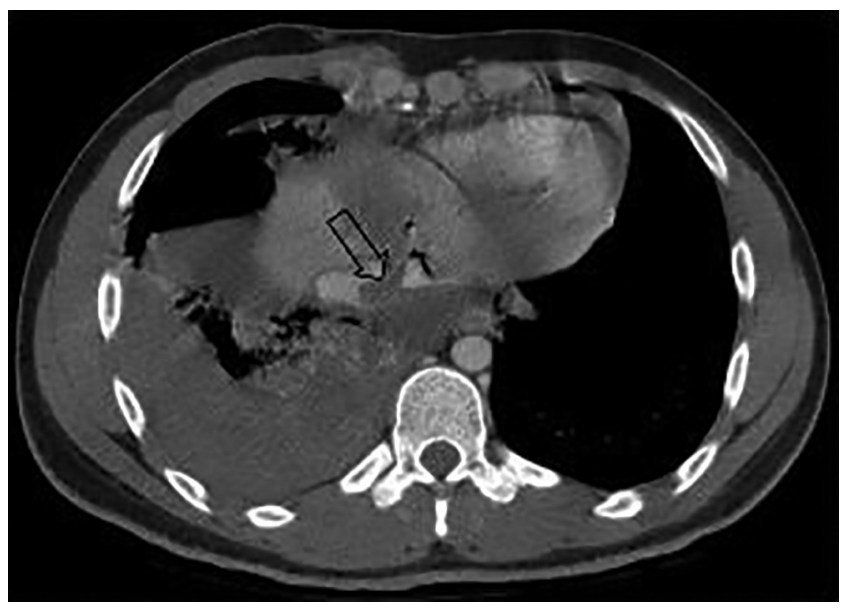

Figure 1 CT demonstrating hepatic rupture. The arrow points to the intra-hepatic IVC tear and thrombus.

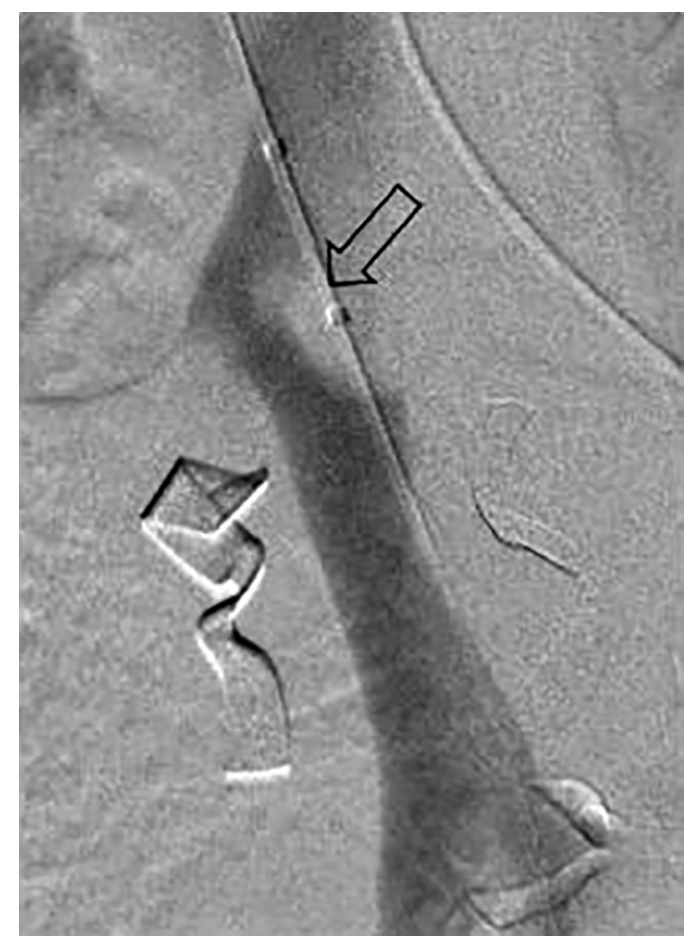

Figure 2 Digital subtraction angiography image demonstrating intra-hepatic IVC thrombus and balloon deflated marking.

$34 \times 100 \mathrm{~mm}^{3}$ thoracic aortic stent graft (SG; Medtronic Valiant ${ }^{\mathrm{TM}}$, USA), which was the only available on shelf SG, was inserted into the RHVC, covering the RHVCI up to the level of the right atrium orifice. In order to prevent iatrogenic Budd-Chiari syndrome, secondary to the occlusion of the hepatic veins drainage to the RHVC by the SG, a covered stent (CS; Gore, Viabahn $9 \times 100 \mathrm{~mm}$ ) was inserted transhepatically, parallel to the SG, like a chimney, draining the right hepatic vein to the right atrium (Figures 3 and 4). 


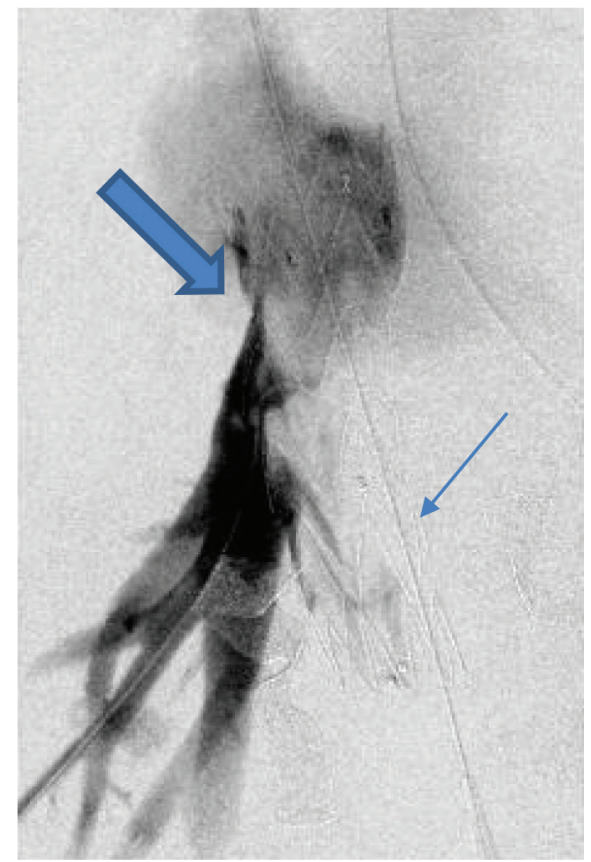

Figure 3 Post-IVC SG implantation digital subtraction angiography via intra-hepatic IV injection. The thin arrow is pointing at the SG and the wide arrow is pointing at the expected obstructed hepatic veins confluence.

The abdomen was temporarily closed, utilizing the vacuum packing technique. A second look laparotomy was done $48 \mathrm{~h}$ after the initial operation, during which the liver packing was removed, and the abdomen was closed.

On the first postoperative day, the patient underwent abdominal CT due to deteriorating hemoglobin counts. The CT revealed that the chimney stents were shown to be functioning properly (Figure 5) and bleeding from the left hepatic artery branches was diagnosed. These branches were angiographically embolized and the bleeding ceased.

Asystole, on the second post-operative day, and symptomatic bradycardia, secondary to complete Atrioventricular $(\mathrm{AV})$ block, were attributed to mechanical irritation of the AV node by the stents, which was confirmed by trans esophageal echo.

The patient was transferred to a cardiac surgery ward, in a level 1 trauma center, where he underwent an urgent operation in order to deal with the chimney stent protrusion into the right atrium. This protrusion also caused an aortic root to the right atrium fistula. The stents were gently shortened, up to just below the inferior vena cava orifice at the right atrium, and the hole in the aortic root was primarily repaired. The patient was transferred to a rehabilitation facility 32 days after the primary trauma.

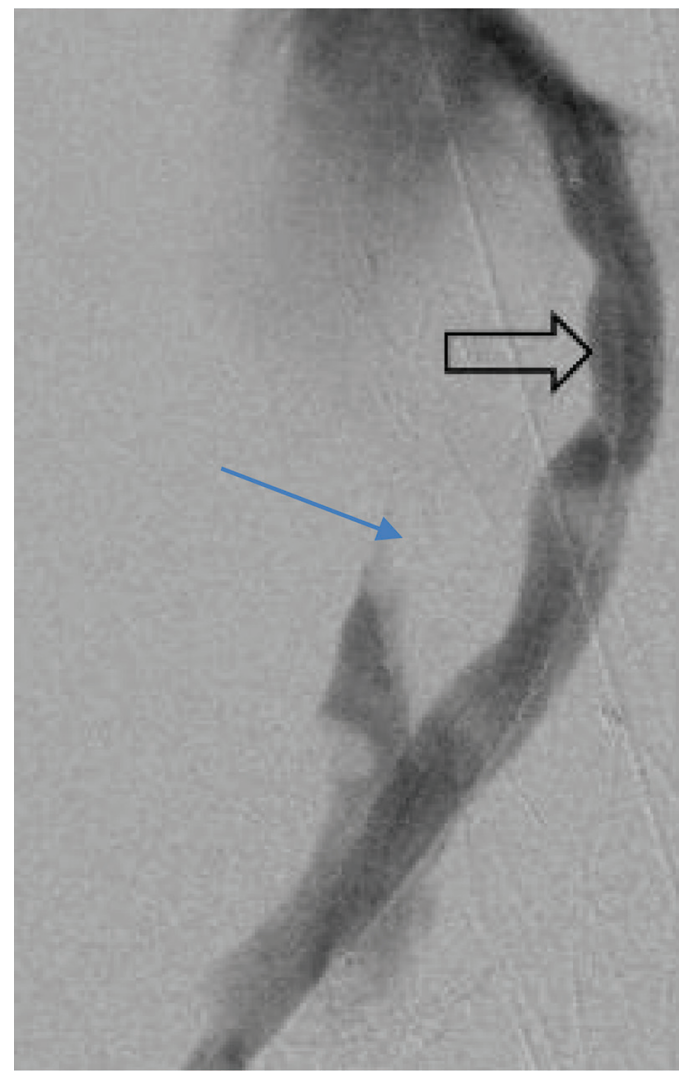

Figure 4 Chimney technique. The thin arrow is pointing at the SG in the RHVC, and the wide arrow is pointing at the Viabahn CS draining the hepatic veins.

\section{DISCUSSION}

Traumatic IVC injuries are among the most challenging and lethal injuries sustained by trauma patients [1]. This is even truer with regard to RHVCI which is associated with even higher mortality rates due to the combination of liver injury and RHVCI [2,3].

Packing is considered as the preferred treatment option. Ligation and/or lateral venography are considered as "second line" maneuvers. The unique location of the RHVCI often necessitates thoraco-abdominal exposure in order to gain proper proximal and distal control over the bleeding vessel and over the abundant bridging veins connecting the liver and the RHVC [4].

Burch's thorough description of the sophisticated techniques and the disappointing experience with the atriocaval shunt concluded that improvement of the treatment techniques should be looked for in the future [5]. The use of an atriocaval shunt is a very challenging maneuver, usually reserved for the most difficult cases for which the preliminary assumption is that it should be used in earlier treatment stages in order to be effective. It is accepted among surgeons dealing with these injuries that the number of published studies regarding the atriocaval shunt technique is larger than the number of survivors $[4,6]$. 


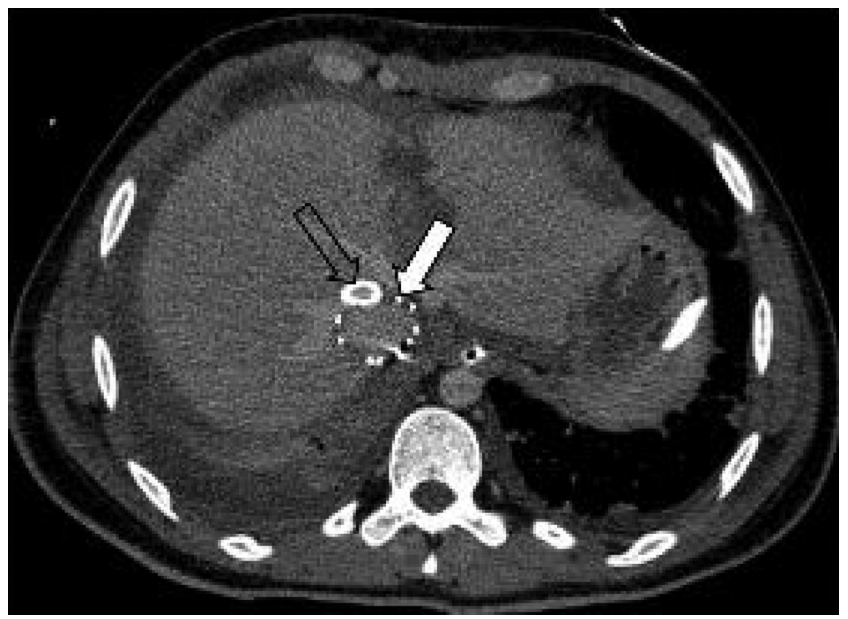

Figure 5 Post-procedure abdominal CT. The white arrow is pointing at the RHVC SG, and the transparent arrow is pointing at Viabahn CS. Both stents are working properly.

Worldwide, most patients with RHVCI are treated in local hospitals lacking hepatobiliary surgeons skilled in dealing with complicated trauma cases. Attempts to explore the RHVC, mobilize the liver, and completely occlude the liver vascularization might be fatal in unexperienced hands.

Rosenthal's description of his small animal study with a new type of shunt, combined with an occluding balloon inserted into the RHVC during the operation, suggested a new approach for treatment. The same concept of excluding the bleeding area by using CS gained popularity several years later [7].

The evolution of endovascular trauma management has recently gained popularity among the vascular and trauma surgery communities, either as a primary definitive treatment modality or as part of a hybrid approach combining endovascular and open treatment tools. Once the only technique used to manage venous injuries, surgical treatment is increasingly being replaced by the endovascular procedure as part of the EVTM concept $[8,9]$.

Endovascular means can be used as an adjunct to the open surgical treatment, using balloon occlusion proximal and distal to the bleeding site, in order to facilitate the exposure [10]. On the other hand, it can be used for definitive treatment using CS to exclude the bleeding area. Total endovascular solution for RHVCI, mainly when done percutaneously, significantly reduces morbidity and mortality.

CS usage for RHVCI carries an inherited problem: an occlusion of the hepatic veins drainage to the RHVC causes secondary Budd-Chiari syndrome. A creative approach should be used to confront this problem, either by creating a splenorenal shunt, an operation rarely done during the current era, or by endovascular means. In-situ laser fenestration of the CS, which might be the preferred treatment option, can be used in selected centers of excellence, with a lot of experience with this technique, mainly in elective cases. However, since this treatment option is not available in our institution and in most of the real world centers, other options, like the one we have used, have to be considered. Using parallel SG in a chimney fashion is a fast and friendly solution [11].

The complications of stent protrusion through the right atrium and secondary AV irritation, as well as the aorto-right atrium fistula, were secondary to stent misplacement. To avoid such complications, adjustable size $\mathrm{CS}$, like the aortic BeGraft (Bentley InnoMed GmbH) whose diameter can be adjusted by balloon inflation, should be used.

\section{CONCLUSION}

Our novel concept of venous chimney procedure for RHVCI, to prevent iatrogenic Budd-Chiari syndrome, highlights the advantages of adopting endovascular arterial treatment concepts to treat extremely complicated venous injuries such as RHVCI. It is obvious that further studies should be conducted in order to assess the short-term as well as the long-term patency of such covered stents in the venous system, and in order to assess the accessory medical treatment that should be given, either with anti-aggregation or anti-coagulation agents, in order to preserve the stent's patency.

\section{Ethics Statement}

(1) All the authors mentioned in the manuscript have agreed to authorship, read and approved the manuscript, and given consent for submission and subsequent publication of the manuscript.

(2) The authors declare that they have read and abided by the JEVTM statement of ethical standards including rules of informed consent and ethical committee approval as stated in the article.

\section{Conflicts of Interest}

The authors declare that they have no conflicts of interest.

\section{Funding}

The author(s) received no financial support for the research, authorship, and/or publication of this article.

\section{REFERENCES}

[1] Buckman RF, Pathak AS, Badellino MM, et al. Injuries of the inferior vena cava. Surg Clin North Am. 2001; 81:1431-47.

[2] Hampton M, Bew D, Edu S, Nicol A, Naidoo N, Navsaria P. An urban trauma centre experience with abdominal vena cava injuries. S Afr J Surg. 2016; 54(1):36-41.

[3] Degiannis E, Velmahos GC, Levy RD, Souter I, Benn CA, Saadia R. Penetrating injuries of the abdominal inferior vena cava. Ann R Coll Surg Engl. 1996;78(6):485-9. 
[4] Perkins ZB, Death HD, Aylwin C, Brohi K, Walsh M, Tai NR. Epidemiology and outcome of vascular trauma at a British Major Trauma Centre. Eur J Vasc Endovasc Surg. 2012;44(2):203-9.

[5] Burch JM, Feliciano DV, Mattox KL. The atriocaval shunt. Facts and fiction. Ann Surg. 1988;207(5):555-68.

[6] Wiencek RG Jr, Wilson RF. Inferior vena cava injuries the challenge continues. Am Surg. 1988;54(7):423-8.

[7] Rosenthal D, Wellons ED, Shuler FW, Levitt AB, Henderson VJ. Retrohepatic vena cava and hepatic vein injuries: a simplified experimental methods of treatment by balloon shunt. J Trauma. 2004;56(2):450-2.

[8] Branco BC, Boutrous ML, DuBose JJ et al. Outcome comparison between open and endovascular management of axillo-subclavian arterial injuries. J Vasc Surg. 2016; 63(3):702-9.

[9] Heldenberg E, Daskal Y, DuBose JJ, Sheizaf G, Aizer $\mathrm{Z}$, Kessel B. Severe venous injury in acute trauma setup - is there a role for endovascular treatment? J Endovasc Resusc Trauma Manag. 2018;2(3):11116.

[10] Galili O, Hebron D, DuBose J, et al. Use of endovascular balloons may simplify Proximal and distal control in complicated vascular trauma. J Endovasc Resusc Trauma Manag. 2017;1(1):39-41.

[11] Tolenaar LJ, Van Keulen WJ, Trimarchi S, Muhs EB, Moll LF, Van Herwaarden AJ. The chimney graft, a systematic review. Ann Surg. 2012;26(7):1030-8. 